\title{
Enhanced optical gain clamping for upstream packet based traffic on hybrid WDM/TDM-PON using Fiber Bragg grating
}

\author{
B. Neto ${ }^{1,2}$, A. Klingler ${ }^{1,3}$, C. Reis ${ }^{1,4}$, R. P. Dionisio ${ }^{1,4,5}$, R. N. Nogueira ${ }^{1,2,6}$, A. L. J. \\ Teixeira $^{1,4,6}$, P. S. André ${ }^{1,2}$ \\ ${ }^{1}$ Instituto de Telecomunicações, Campus Universitário de Santiago, 3810-193 Aveiro, Portugal \\ ${ }^{2}$ Departamento de Física, Campus Universitário de Santiago, 3810-193 Aveiro, Portugal \\ ${ }^{3}$ Faculty of Engineering and Computer Science of Ulm University, Albert-Einstein-Allee 39 \\ 89081 Ulm, Germany \\ ${ }^{4}$ Departamento de Electrónica, Telecomunicações e Informática, Campus Universitário de Santiago, 3810-193 \\ Aveiro, Portugal \\ ${ }^{5}$ Escola Superior de Tecnologia de Castelo Branco (ESTCB), Avenida do empresário, 6000-767- Castelo Branco, \\ Portugal \\ ${ }^{6}$ Nokia-Siemens Networks (NSN), Rua Irmãos Siemens, 12720-093 Amadora, Portugal \\ "Corresponding author: bneto@av.it.pt
}

\begin{abstract}
In this paper, we propose a method to mitigate the temporal power transients arising from Erbium doped fiber amplifiers (EDFAs) on packeted/bursty scenario. The technique, applicable on hybrid WDM/TDMPON for extended reach, is based on a low power clamping provided by a distributed feedback (DFB) laser and a fiber Bragg grating (FBG). An improvement in the data signal Q factor was achieved keeping the clamping control signal with a low power, accompanied by a maximum reduction in the gain excursion of $1.12 \mathrm{~dB}$.
\end{abstract}

(C)2010 Optical Society of America

OCIS codes: (060.4259) Networks, packet-switched, (060.2320) Fiber optics amplifiers and oscillators, (060.2410) Fibers, erbium.

\section{References and links}

1. K. Ennser, M. Zannin, S. Taccheo, "Extending Reach of Passive Optical Networks through Optical Amplification", in International Conference on Transparent Optical Networks, ICTON 2009, Azores, paper Tu.D5.1, 2009.

2. J. A. Lázaro, J. Prat, P. Chanclou, G. M. Tosi Beleffi, A. Teixeira, I. Tomkos, R. Soila, and V. Koratzinos, "Scalable Extended Reach PON," in Optical Fiber Communication Conference and Exposition and The National Fiber Optic Engineers Conference, OFC/NFOEC 2008, paper OThL2.

3. Y. Fukada, K. Suzuki, H. Nakamura, N. Yoshimoto, M. Tsubokawa, "First demonstration of fast automatic gain control (AGC) PDFA for amplyfyng burst-mode PON upstream signal", European Conference on Optical Communication, ECOC 2008, paper We.2.F.4.

4. Y. Sun, G. Luo, J. L. Zyskind, A. A. M. Saleh, A. K. Srivastava, J. W. Sulhoff, "Model for gain dynamics in Erbium-doped fibre amplifiers", IEE Electron Lett 32, (1996) 1490-1491.

5. Yoshinari Awaji, Hideaki Furukawa, Naoya Wada, Peter Chan, Ray Man, "Mitigation of the transient response of Erbium doped fiber amplifiers for burst traffic high speed optical packets", in Conference on Lasers and Electro-Optics, CLEO 2007, paper JTuA133.

6. Yoshinari Awaji, Hideaki Furukawa, Nayoa Wada, Eddie Kong, Peter Chan, Ray Man, "Guidelines for amplification of optical packets in WDM environment regarding impact of transient response of Erbiumdoped fiber amplifier", Computer Networks 52 (2008) 2087-2093.

7. Y. Liu, C. W. Chow*, C. H. Kwok, H. K. Tsang and Chinlon Lin,"Optical Burst and Transient Equalizer for $10 \mathrm{~Gb} / \mathrm{s}$ Amplified WDM-PON, in Optical Fiber Communication Conference and Exposition and The National Fiber Optic Engineers Conference, OFC/NFOEC 2008, paper OthU7.

8. G. Sacchi, S. Sugliani, A. Bogoni, F. Di Pasquale, R. Di Muro, R. Magri, G. Bruno, F. Cavaliere, "Design and experimental characterization of EDFA-based WDM ring networks with free ASE light recirculation and link control for network survivability", J. Ligthwave Technol 23, (2005), $1170-1181$. 
9. A.V. Tran; Chang-Joon Chae; R.S. Tucker; Yang Jing Wen, "EDFA transient control based on envelope detection for optical burst switched networks", Photon Technol Lett 17, (2005) 226 - 228.

10. Alberto Bononi and Leslie A. Rusch, "Doped-Fiber Amplifier Dynamics: A System Perspective," J. Lightwave Technol. 16, 945- (1998).

11. B. Neto, C. Reis, R. P. Dionísio, J. M. Ferreira, J. A. Lazaro, G. Tosi-Beleffi, A. N. Pinto, R. Nogueira, A. Teixeira, J. Prat, P. S. André, "Assessment and mitigation of EDFA gain transients in hybrid WDM/TDM PON in the presence of packet based traffic", Special issue on Next Generation Optical Access, IET Journal of Optoelectronics (to be published in mid 2010).

\section{Introduction}

Hybrid wavelength division multiplexing/ time division multiplexing passive optical networks (WDM/TDM-PONs) are currently under intensive research in order to become possible candidates for the next-generation of high-bandwidth $(\sim 10 \mathrm{~Gb} / \mathrm{s})$ optical access networks, due to their costeffective user-shared facilities. To provide more cost-insensitive services, system designers need to improve the power budget by extending the network reach and increasing the splitting ratios of the nodes [1]. As an example, the recently proposed Scalable Advanced Ring-based passive Dense Access Network Architecture (SARDANA) seeks to establish an optical passive transparent infrastructure over a dense extended-range area, capable of supporting future high broadband services [2]. The use of optical amplifiers, such as Erbium Doped Fiber Amplifiers (EDFAs), can increase the system power budget significantly, but there are issues to be accounted for in the implementation of amplified WDM/TDM-PONs, namely in the upstream traffic, where the signal is bursty. In that case, EDFA gain transient and gain spectrum tilting will make the optical bursts exceed the dynamic range of receivers and induce power penalty.

Some solutions to mitigate optical amplifiers transients for burst mode PON were successfully demonstrated and are based on a combination of techniques, such as linear gain control, gain clamping and fast automatic gain control [3-4]. Additionally, passive mitigation approaches have also been proposed, such as the use of Erbium doped fibre (EDF) with a large active area of $\mathrm{Er}^{3+}$ ions [5-6] and also transient equalizers based on silicon-on insulators [7].

In the optical domain, the idea of re-circulating amplified spontaneous emission (ASE) as a clamping mechanism in a feed-back loop was presented in the context of WDM ring networks [8]. Hybrid electronic/optical techniques to achieve transient mitigation are valuable such as the approach presented in [9], where a packet envelope technique is used to generate an inverted modulation on a control signal and therefore promoting the mitigation of the transients. The use of distributed feedback (DFB) laser signal to clamp the EDFA gain in a WDM/TDM-PON was also proposed, using a feedback scheme that employs the downstream channel to clamp the gain of the upstream packet channel [10]. The approach proposed in this paper is based on gain clamping by means of a DFB signal and the use of a Fiber Bragg Grating (FBG) to enhance the clamping effect by re-injecting the signal backwardly the clamping. This approach has the merit of increasing the optical signal to noise ratio (OSNR) in comparison to clamping methods that use re-circulating ASE and is cost efficient since the control signal can be supplied by part of the downstream channel, since the reported clamping powers assume low values $[-25.74,-1.67] \mathrm{dBm}$. The work presented here compares the effect of enhanced clamping with a FBG and simple clamping by presenting Q-factor and gain excursion results, suggesting its application for future WDM/TDM-PON architectures.

The paper is organized as follows: in section 2, we described the proposed mitigation concept and its feasibility in the concept of WDM/TDM PON. The implemented experiment used to verify this technique in a lab environment is presented in section 3 . The obtained main results and further discussion are presented on section 4 . Finally, in section 5 , the conclusions are drawn. 


\section{Transient mitigation concept}

The idea of this technique is to use the intrinsic characteristics of bidirectional traffic existing in PON as a transient mitigation mechanism for the packeted/bursty upstream traffic. In such architecture, instead of using re-circulating ASE that degrades the OSNR, the downstream channel itself acts as a clamping signal. Since we presume that its power is possibly low due to the PON operation and that by this reason the clamping on the EDFA transient could be insufficient, a FBG was inserted in order to enhance the clamping. Unlike conventional clamping mechanisms, the proposed method does not use feed-back.

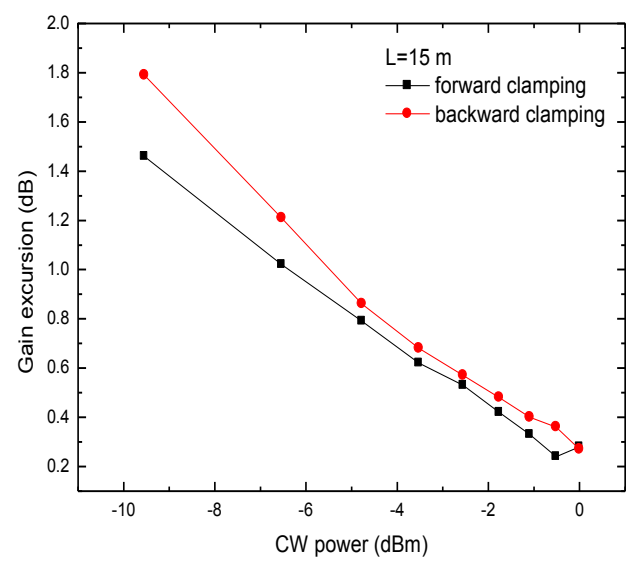

Fig. 1- Packet gain excursion as function of control signal power for a EDF with $15 \mathrm{~m}$. (black squares) Forward clamping, (red circles) backward clamping.

Although the scheme suggests backward clamping for a matter of simplicity, forward clamping is also feasible. To infer the efficiency of forward and backward clamping, some simulations were carried on. They compute the packet gain excursion by implementation of Bononi et al dynamical model for EDFA [10]. The results obtained for a span of $15 \mathrm{~m}$ of EDF using $20 \mu$ s packets are plotted on Fig. 1. On looking at the results, it is noticeable that despite the forward clamping is more efficient on mitigating the transient; the method converges when the clamping power is increased.

\section{Experimental assessment}

To demonstrate experimentally the proposed mitigation approach, we implemented the setup depicted in Fig. 2 for a forward clamping scheme.

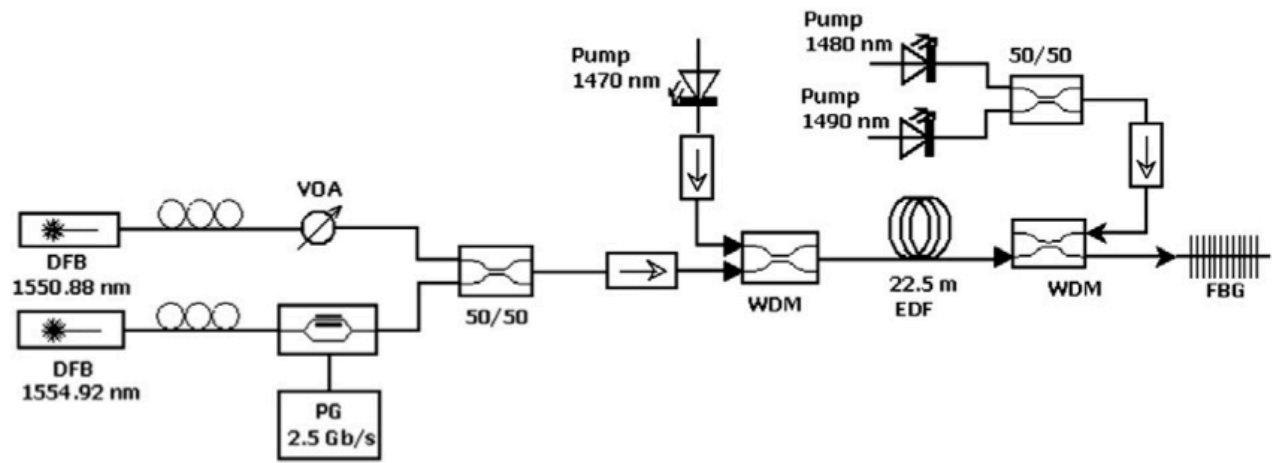

Figure 2- Scheme of the implemented experimental setup. 
Although, some of the used equipments are not cost efficient for PON, the experiments serves the purpose to demonstrate the technique in a laboratorial environment. It consists of two DFB lasers peaking at $1554.92 \mathrm{~nm}$ and $1550.80 \mathrm{~nm}$ with optical powers of $13.0 \mathrm{dBm}$ and $3 \mathrm{dBm}$, respectively, followed by polarizations controllers. The laser at $1554.92 \mathrm{~nm}$ works as the upstream data signal and is optically modulated by a Mach-Zehnder operating at $2.5 \mathrm{~Gb} / \mathrm{s}$ with an extinction ratio of $14.4 \mathrm{~dB}$. The data are programmable packets with a duration of 26 $\mu$ s (pseudo random bit sequence- PRBS of length $2^{16}-1$ ) and an idle time equal to $20 \mu \mathrm{s}$ (50000 bits), produced by a BERT (Agilent N4901B). The signal at $1550.80 \mathrm{~nm}$ works in continuous wave $(\mathrm{CW})$ and represents the clamping control signal, being its power adjusted by a variable optical attenuator (VOA). The two signals are brought together by a $50 / 50$ coupler and injected into a bidirectionally pumped highly EDF (Thorlabs ER-4/125) with a span of $22.5 \mathrm{~m}$. The average inputted power of the data signal is $-7.0 \mathrm{dBm}$. The EDF pumps are three diode lasers peaking at $1470 \mathrm{~nm}, 1480 \mathrm{~nm}$ and $1490 \mathrm{~nm}$ with a total injected power of $24.0 \mathrm{dBm}$. Two WDM couplers are used to join signals and pumps as well as three isolators to protect the sources. After the amplification stage, a FBG with a rejection of $27.0 \mathrm{~dB}$ and an optical bandwidth, $\Delta \lambda$, equal to $0.81 \mathrm{~nm}$ is inserted in order to reflect the clamping control signal backwardly into the EDF. To analyze the temporal evolution of the upstream data packets, a PIN (HP-11982A) with responsivity of $0.7 \mathrm{~A} / \mathrm{W}$ and an (Agilent Infinium 86100A) oscilloscope were used. The gain excursions of packets as well as the Q factor were experimentally analyzed.

For the most penalizing situation, with the clamping control signal off (unclamped), the data signal was visualized on the oscilloscope, being the extinction ratio and Q factor measured. The measured $\mathrm{Q}$ factor values are $4.74 \mathrm{~dB}$ and $3.04 \mathrm{~dB}$ for back-to-back and after the amplification stage, respectively. The gain excursion for the late situation is $2.16 \mathrm{~dB}$.

In order to emphasize the effect of the FBG in the proposed mitigation setup, we compare the results obtained with (simple clamping) and without (enhanced clamping) the FBG and varying the clamping control signal power $-25.74 \mathrm{dBm}$ to $-1.67 \mathrm{dBm}$, being the $\mathrm{Q}$ factor measured.

\section{Results and discussion}

The use of a control signal is able to clamp the gain whenever its power is high enough, as stated from the square plot on Fig. 3, where the gain excursion is plotted as a function of the clamping control input power for the implemented situations: simple clamping and enhanced clamping. Hence, a decrease on the gain excursion is observed when the control power is increased. However, the use of a FBG with Bragg wavelength coincident with the control signal is able to re-inject it backwardly into the fiber, enhancing the clamping while keeping the same amount of power. A decrease on the gain excursion is observed, when the control power is increased. Both results were linearly fitted. For the simple clamping, the linear fit provides a slope equal to $-0.047 \mathrm{~dB} / \mathrm{dBm}$, the enhanced clamping data has a slope equal to $0.036 \mathrm{db} / \mathrm{dBm}$. In the latter case, the gain excursion decreases $1.12 \mathrm{~dB}$, when the control power is equal to $-25.7 \mathrm{dBm}$. 


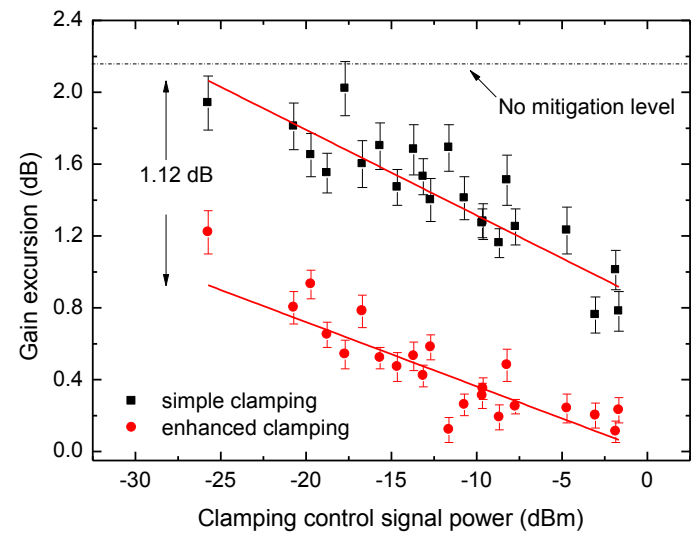

Fig. 3- Packet gain excursion as function of control signal power. Continuous lines are linear fits. (squares) - Simple clamping, adjusted $\mathrm{r}$ square $=0.79$; (circles) enhanced clamping, adjusted $\mathrm{r}$ square $=0.73$. The dashed line stands for the gain excursion level for the unclamped situation.

The clamping effect is also visible in the Q-factor that exhibits an improvement with the control signal power. These results are displayed in Fig. 4, where the $\mathrm{Q}$ factor as function of the control signal power is plotted for simple clamping and enhanced clamping. On looking at the results, we verify that the simple clamping shows two distinct regimes. The first one, observable for low powers (until $-15.7 \mathrm{dBm}$ ), demonstrates that the $\mathrm{Q}$ factor remains stable and the clamping is not assessable. However, from that power value the Q-factor starts to grow linearly with the clamping control signal power. The interpretation of this behavior is based on the idea that, bellow a predefined value of power, the effect of the clamping signal is negligible. Above this value, the clamping has an effect on decreasing the gain excursion and the Q-factor increases. The same behavior is not observable for the enhanced clamping in which the $\mathrm{Q}$ factor remains almost constant with an average value of $4.71 \mathrm{~dB}$ in the range of the inputted control powers. By decreasing the gain excursion over a threshold value the Qfactor was definitely increased. It must be noted that, the use of the FBG was able to improve the $\mathrm{Q}$ factor to a value close to that of back-to-back values.

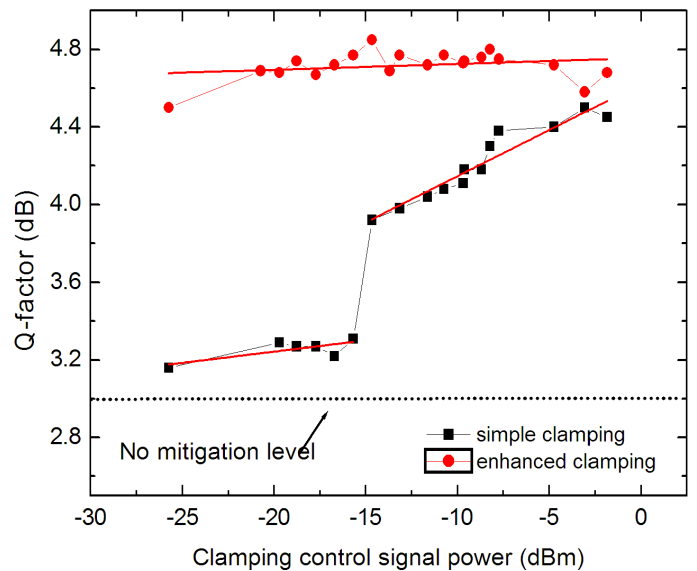

Fig. 4- Q factor at the receiver as a function of the clamping control signal power. (squares) - Simple clamping and (circles) enhanced clamping. The dashed line stands for the Q-factor level for unclamped situation. 
The dynamics in terms of gain excursion are determined by the packet duration and idle times. By increasing idle times, the EDFA restores the initial conditions more effectively and thus the gain excursion on the next packet envelope that income the EDF is larger. By increasing the packet duration the depletion effect on the excited Erbium ions population is also more significant. If the system is not given enough time to restores the initial conditions, the next incoming packet with experience less gain and consequently less gain excursion. So, the results in terms of gain excursion are a combination of these factors. However, the exponential dependency of the filling and depletion effects of the Erbium excited state population imposes a stabilization effect $[10,11]$ so asymptotic behaviors can be settled.

Hence, although, the method was analyzed by considering a specific situation of packet and idle times, it is expected that the results complying other traffic characteristics will only differ in terms of the necessary clamping powers. Thus, the mitigation technique can be seemingly extrapolated to different types of packet traffic.

\section{Conclusions}

We present an enhanced clamping method based on the use a control signal and a FBG. The method is feasible in the context WDM/TDM-PON for upstream bursty traffic to mitigate EDFA transients and can be extrapolated for other contexts such as WDM-PON. An experimental assessment was implemented for a pumped EDF using an input packet channel at $-7.0 \mathrm{dBm}$. In that situation, the output packet exhibits a gain excursion of $2.16 \mathrm{~dB}$, using very low clamping control signal power $(-25.74 \mathrm{dBm})$ and a FBG reduces the gain excursion in $1.12 \mathrm{~dB} \mathrm{~dB}$ while increasing the $\mathrm{Q}$ factor to $4.71 \mathrm{~dB}$. Although the experimental setup does not suggest any specific architecture, it can easily be adapted to WDM/TDM-PON. In that practical application the use of a portion of the downstream signal could work as a clamping control signal to clamp the upstream packet transients in a way that is affordable and preserves the passiveness of the network.

\section{Acknowledgement}

This work was supported by the POSC program, by the European Union FEDER fund and by the Portuguese scientific program. B. Neto gratefully acknowledges the $\mathrm{Ph}$. D. grant financed by FCT (SFRH/BD/28904/2006). 